\title{
Prediction of daily contagions of CoViD-19-SARS- CoV-2 from August 24 to July 31, 2021 in Apulia, a region of Italy, by using a New Epidemiologic Fractal Model.
}

\section{Calogero Rinzivillo}

University Catania

\section{Sergio Conte}

School of Advanced International Studies on Applied Theoretical and non Linear Methodologies of Physics

\section{Francesco Casciaro}

School of Advanced International Studies on Applied Theoretical and non Linear Methodologies of Physics

\section{Renato Bernardini}

University Catania

\section{Elena Benigni}

WONDER (World Organization for Noetic, Dimensional and Evolutionary Research)

Ines Paola Monte

University Catania

\section{Ferda Kaleagasioglu}

Istinye University

\section{Fang Wang}

Hunan Agricultural Institute

\section{Cengiz Mordeniz}

Tekirdag Namik Kemal University

\section{Giuseppe Liberti}

Cannizzaro Hospital

Elio Conte ( $\nabla$ elio.conte@fastwebnet.it )

School of Advanced International Studies on Applied Theoretical and non Linear Methodologies of Physics

\section{Short Report}

Keywords: epidemiology, CoVid 19, fractal model 
Posted Date: October 30th, 2020

DOI: https://doi.org/10.21203/rs.3.rs-98910/v1

License: (c) (i) This work is licensed under a Creative Commons Attribution 4.0 International License. Read Full License 


\title{
Prediction of daily contagions of CoViD-19 - SARS-CoV-2 from August 24 to July 31, 2021 in Apulia , a region of Italy, by using a New Epidemiologic Fractal Model.
}

\author{
Calogero Rinzivillo ${ }^{(1)}$, Sergio Conte ${ }^{(2)}$, Francesco Casciaro ${ }^{(2,3)}$, Renato Bernardini ${ }^{(4)}$, Elena Benigni ${ }^{(5)}$, Ines Paola \\ Monte $^{(6)}$, Ferda Kaleagasiogl $\mathrm{u}^{(2,7)}$, Fang Wang ${ }^{(8)}$, Cengiz Mordeniz ${ }^{(2,9)}$, Giuseppe Liberti ${ }^{(10)}$, Elio Conte ${ }^{(2)}$ \\ ${ }^{(1)}$ Department of Medical-Surgical Sciences, Organs Transplantation and Advanced Technologies \\ (DGFI@unict.it) -Policlinic of University of Catania, Italy. \\ ${ }^{(2)}$ School of Advanced International Studies on Applied Theoretical and non Linear Methodologies of \\ Physics, Bari, Italy. \\ (3) U.O.C. "Medicine and Surgery of Urgency", "Sacro Cuore di Gesù" Hospital in Gallipoli (Le) \\ ${ }^{(4)}$ Department of Biomedical and Biotechnological Sciences, Section of Pharmacology, University of Catania \\ School of Medicine. \\ ${ }^{(5)}$ WONDER (World Organization for Noetic, Dimensional and Evolutionary Research), Rome, Italy. \\ ${ }^{(6)}$ Department of General Surgery and Medical-Surgical Specialities- University of Catania \\ ( (7) Department of Pharmacology and Clinical Pharmacology, Istinye University Topkapı Campus, Maltepe \\ Neighbourhood, Teyyareci Sami Street, Building no.3, Zeytinburnu, İstanbul, Turkey, 34010 \\ (8) Hunan Agricultural University, Changsha, China' \\ (9) Department of Anesthesiology, Intensive care and pain Medicine, Tekirdag Namik Kemal University, \\ Turkey \\ ${ }^{(10)}$ CoViD-19 regional commissioner - Division of Infective Diseases, Cannizzaro Hospital, Catania.
}

\begin{abstract}
We study a new fractal model for prediction of contagions of CoViD-19 (Sarbeco Betavirus SARSCoV-2) from August 24, 2020 to July 31, 20201 in Apulia that is a region of Italy. We obtain that the time of the peak is estimated to be at December 7, 2020 and the number of contagions will be about 575 cases.
\end{abstract}

\section{Introduction}

The aim of the present work is to effect a prediction of the contagions of SARS-CoV-2 in Apulia in the period from August 24, 2020 to July 31, 2020.In the work we use the method of the fractal analysis, fitting a new fractal model following the performance that was used previously from Ziff and Ziff [1] during the contagions in China. The current prediction, effected by such new model introduced by us, is that the size of the epidemic will be about 575 cases of contagions in Apulia and the time of peak will be about December 7, 2020 on the general plane, depending instead the actual size of the process from the respect or an increase o decrease of the prevention measures that are fixed from the governing bodies.

\section{Materials and Methods}

The power-law (fractal) behavior has been postulated and applied in epidemic studies of Corona Virus disease in China. It is related to the properties of the networks that carry out the propagation of the disease. Vazquez [2] developed a network model, Anna L. Ziff and Robert M. Ziff [1] applied a fractal 
behavior model in contagions in China. The daily number of new contagions cases, $n(t)$, in an epidemic followed a power-law with an exponential cutoff

$n(t)=k t^{\gamma} \exp \left(-t / t_{0}\right)$

This model was used in our previous estimations $[3,4,5]$. We have modified such model introducing an additive linear term $C(n)$. Therefore, the model that we adopt is the following

$n(t)=k t^{\gamma} \exp \left(-t / t_{0}\right)+C(n)$

where $C(n)$ is a linear parameter that is established by the fitting operation.

\section{Results}

We applied the previous model for the contagions of SARS-CoV-2 in Apulia. The parameter values that we estimate are the follows

$k=0.000385$

$\gamma=3.8711$

$t_{0}=27.0511$

$C(n)=45.00$

They confirm that we are in presence of a fractal regime given by the non-integer value of $\gamma$.The value $\gamma \mathrm{t}_{0}$ represents the Time of the Peak. The results are in Figure 1. The time explored is from August 24, 2020 to July 31, 2021. It is seen that the time peak is about the December 7, 2020 with a total of contagions of about 575 cases.

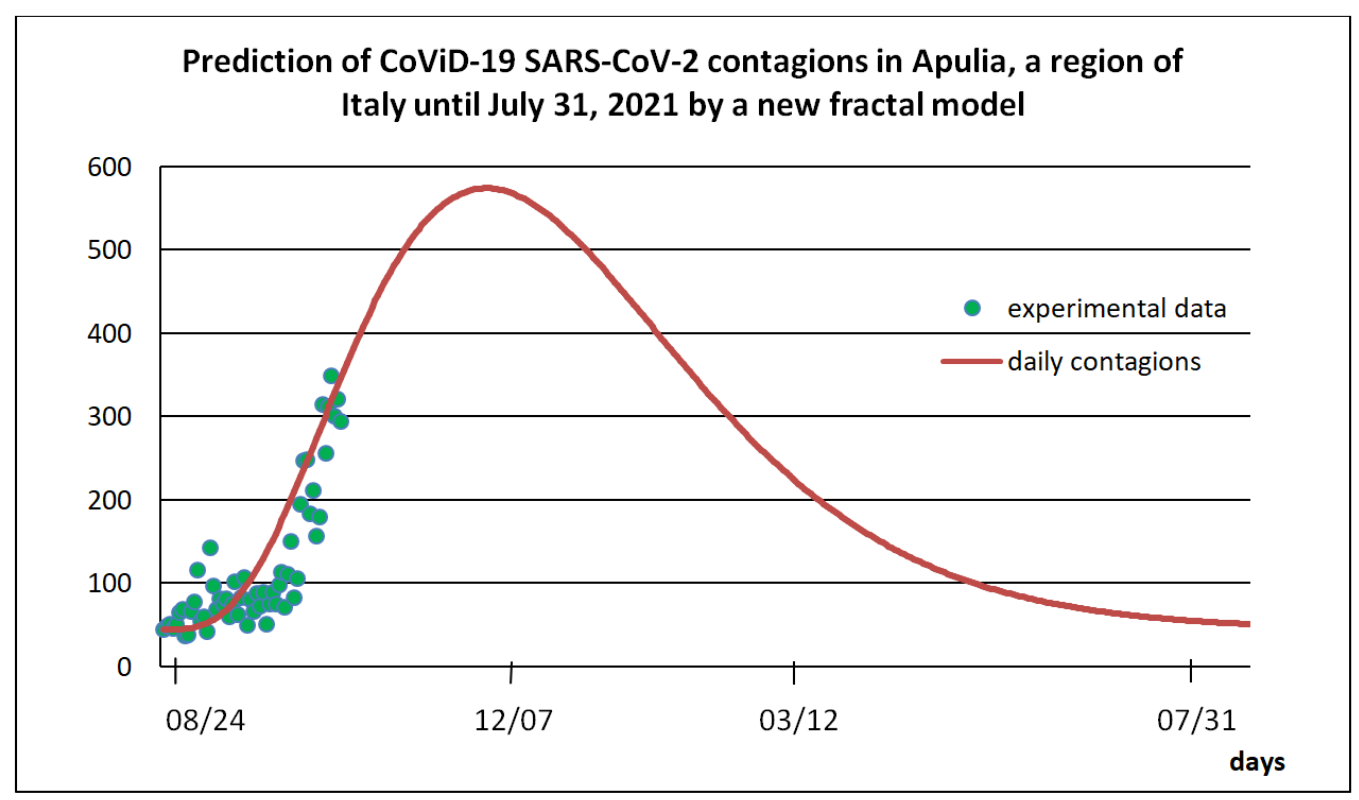

Figure 1 
We calculated also the correlation existing daily between the number of contagions and the swabs made. The results that follow, indicate that such correlation exists with two stars .

\begin{tabular}{|l|l|}
\hline Number of XY Pairs & 13 \\
\hline Pearson $r$ & 0,6991 \\
\hline $95 \%$ confidence interval & 0.2408 to 0.9025 \\
\hline P value (two-tailed) & 0,0078 \\
\hline P value summary & $* *$ \\
\hline Is the correlation significant? (alpha $=0.05)$ & Yes \\
\hline R squared & 0,4888 \\
\hline
\end{tabular}

\section{References}

[1] Anna L. Ziff and Robert M. Ziff, Fractal kinetics of COVID-19 pandemic (with update 3/1/20), medRxiv preprint doi: https://doi.org/10.1101/2020.02.16.20023820.

[2] Alexei Vazquez. Polynomial growth in age-dependent branching processes with diverging reproductive number. Physical Review Letters, 96(3):038702, January 2006. ISSN 0031-9007, 1079-7114.doi: 10.1103/ Phys. Rev. Lett.96.038702. URL http://arxiv.org/abs/cond-mat/0505116. arXiv: cond-mat/0505116.

[3] Calogero Rinzivillo, Sergio Conte, Ferda Kaleagasioglu, Fang Wang, Alberto Foletti, Elio Conte, The Basic Indications and the Clinical Implications of Being the SARS Covid-19 a multifractal. vixra [v1] 2020-04-07

[4] Calogero Rinzivillo, Sergio Conte, Ferda Kaleagasioglu, Fang Wang, Alberto Foletti, Elio Conte, Prediction of Daily Contagions of Covid-19 from March 01 to May 19 in Italy. vixra[v1] 2020-03-17

[5] Calogero Rinzivillo, Sergio Conte, Ferda Kaleagasioglu, Fang Wang, Alberto Foletti, Cengiz Mordeniz, Elio Conte, New Prediction of Sars-Covid-19 in Italy. vixra [v1] 2020-04-24 
Figures

Prediction of CoViD-19 SARS-CoV-2 contagions in Apulia, a region of Italy until July 31, 2021 by a new fractal model

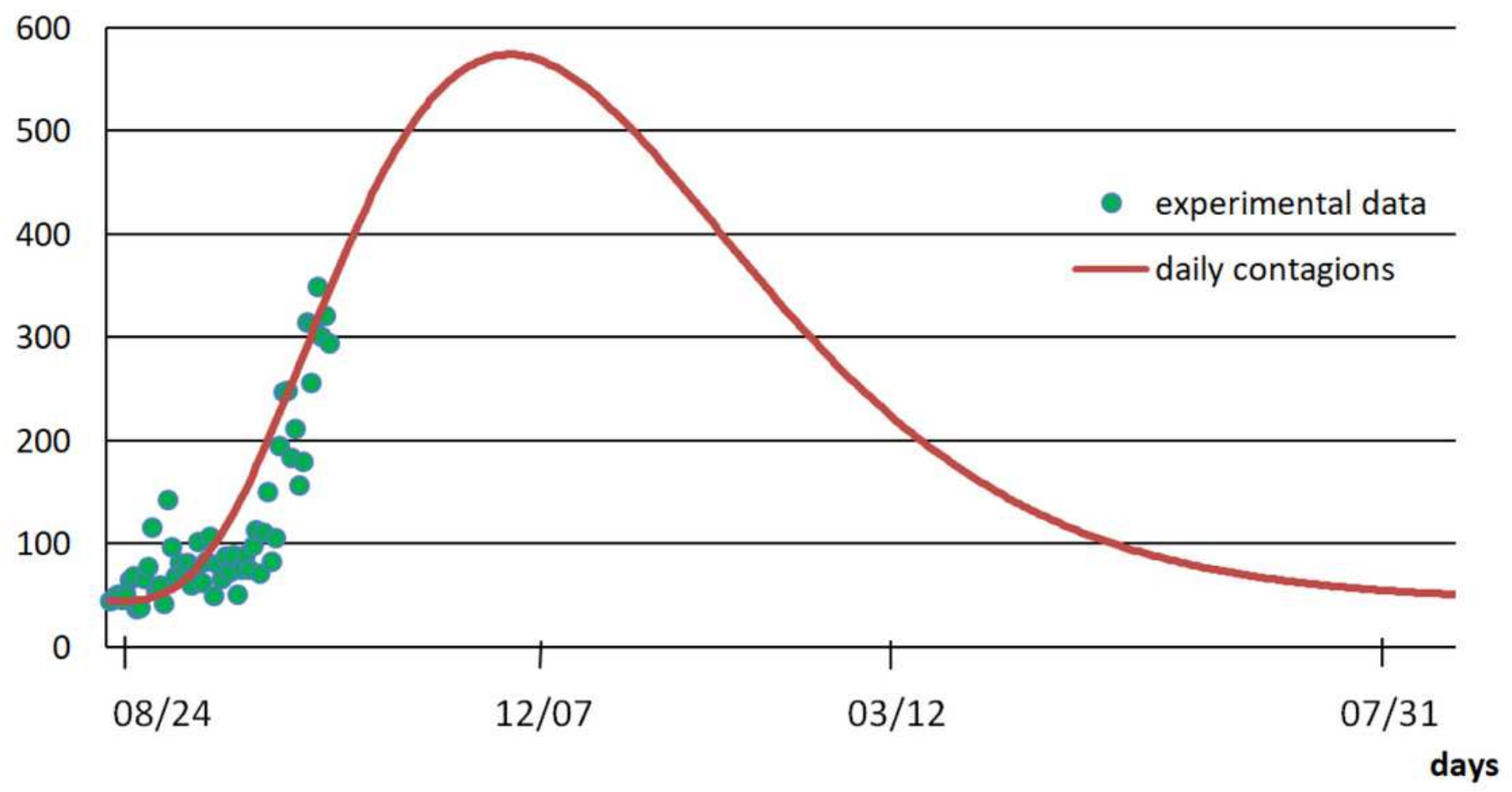

Figure 1 